\title{
An abstract nonlinear second order differential equation
}

\author{
by JAN BOCHENEK (Kraków)
}

\begin{abstract}
By using the theory of strongly continuous cosine families of linear operators in Banach space the existence of solutions of a semilinear second order differential initial value problem (1) as well as the existence of solutions of the linear inhomogeneous problem corresponding to (1) are proved. The main result of the paper is contained in Theorem 5.
\end{abstract}

1. Introduction. We consider the abstract semilinear second order initial value problem

$$
\left\{\begin{array}{l}
\frac{d^{2} u}{d t^{2}}=A u+f\left(t, u, \frac{d u}{d t}\right), \quad t \in(0, T] \\
u(0)=u_{0}, \quad \frac{d u}{d t}(0)=u_{1},
\end{array}\right.
$$

where $A$ is a linear (possibly unbounded) operator from a real Banach space $X$ into itself, $u$ is a mapping from $\mathbb{R}$ to $X, f$ is a nonlinear mapping from $\mathbb{R} \times X \times X$ into $X$ and $u_{0}, u_{1} \in X$. In this note we try to give a systematic and general treatment of the problem of existence, uniqueness and smoothness of solutions of (1). The pioneering work on this problem was done by Segal [3]. Here we extend some results by C. C. Travis and G. F. Webb [4]. In particular, we consider the classical solutions of (1) under more general hypotheses on the nonlinear term $f$ than in [4]. Our main tool is the theory of strongly continuous cosine families of linear operators in Banach space. The basic ideas and results of this theory can be found in [4].

2. Preliminaries. Let $A$ be the linear operator defined in Section 1 . We make the following assumption on $A$.

$\left(\mathrm{Z}_{1}\right) \quad A$ is the infinitesimal generator of a strongly continuous cosine family $\{C(t): t \in \mathbb{R}\}$ of bounded linear operators from $X$ into itself.

1985 Mathematics Subject Classification: Primary 34K30. 
Recall that the infinitesimal generator of a strongly continuous cosine family $C(t)$ is the operator $A: X \supset D(A) \rightarrow X$ defined by

$$
A x:=\left.\left(d^{2} / d t^{2}\right) C(t) x\right|_{t=0}, \quad x \in D(A),
$$

where

(3) $D(A):=\{x \in X: C(t) x$ is twice continuously differentiable in $t\}$.

Let

$E:=\{x \in X: C(t) x$ is once continuously differentiable in $t\}$.

It is known (see [4, Proposition 2.2]) that $D(A)$ is dense in $X$ and $A$ is a closed operator in $X$.

We define the associated sine family $S(t), t \in \mathbb{R}$, by

$$
S(t) x:=\int_{0}^{t} C(s) x d s, \quad x \in X, t \in \mathbb{R} .
$$

From assumption $\left(\mathrm{Z}_{1}\right)$ it follows (see $[4,(2.11)$ and $\left.(2.12)]\right)$ that there are constants $M \geq 1$ and $\omega \geq 0$ such that

$$
\|C(t)\| \leq M e^{\omega|t|} \quad \text { and } \quad\|S(t)\| \leq M e^{\omega|t|} \quad \text { for } t \in \mathbb{R} .
$$

Remark that $S(t) X \subset E$ and $S(t) E \subset D(A)$ for $t \in \mathbb{R},(d / d t) C(t) x=$ $A S(t) x$ for $x \in E$ and $t \in \mathbb{R}$, and $\left(d^{2} / d t^{2}\right) C(t) x=A C(t) x=C(t) A x$ for $x \in D(A)$ and $t \in \mathbb{R}$ (see $[4,(2.17)-(2.19)]$ ).

For $x \in X$ and $s, r \in \mathbb{R}$, we have (see [4])

$$
\begin{gathered}
\int_{s}^{r} S(t) x d t \in D(A), \\
A \int_{s}^{r} S(u) x d u=[C(r)-C(s)] x .
\end{gathered}
$$

Note that the adjoint operator $A^{*}: X^{*} \rightarrow X^{*}$ is well defined, for $\overline{D(A)}=X$.

We make the following assumption on $A^{*}$.

$\left(\mathrm{Z}_{2}\right) \quad$ The adjoint operator $A^{*}$ is densely defined in $X^{*}$, i.e. $\overline{D\left(A^{*}\right)}=X^{*}$.

Lemma 1. Suppose $\left(\mathrm{Z}_{1}\right)$ and $\left(\mathrm{Z}_{2}\right)$. Let $g:[0, T] \rightarrow X$ be a Lipschitzian mapping with Lipschitz constant $L>0$. Then the formula

$$
v(t):=\int_{0}^{t} S(t-s) g(s) d s, \quad t \in[0, T],
$$

defines a function from $[0, T]$ into $D(A)$. 
Proof. Let $t \in[0, T]$. For every $n \in \mathbb{N}$ set

$$
u_{n}(t)=\int_{0}^{t} S(t-s) g_{n}(s) d s,
$$

where

$$
g_{n}(s):=\sum_{i=0}^{n-1} g\left(t_{i}\right) \chi_{B_{i}}(s), \quad s \in[0, t]
$$

$B_{i}=\left[t_{i}, t_{i+1}\right)$ for $i=0,1, \ldots, n-2 ; B_{n-1}=\left[t_{n-1}, t_{n}\right], t_{i}=i t / n, i=$ $0,1, \ldots, n$, and $\chi_{B_{i}}$ denotes the characteristic function of $B_{i}$.

Clearly $\left\{g_{n}\right\}$ converges uniformly on $[0, t]$ to $g$ (in the norm topology) and $\left\{u_{n}(t)\right\}$ converges in norm to $v(t)$. By virtue of $(6)$ and $(9),\left\{u_{n}(t)\right\} \subset D(A)$. By (7) we have

$$
\begin{aligned}
A u_{n}(t)= & \sum_{i=0}^{n-1}\left[C\left(t-t_{i}\right)-C\left(t-t_{i+1}\right)\right] g\left(t_{i}\right) \\
= & {[C(t)-I] g\left(t_{0}\right)+C\left(t-t_{1}\right)\left[g\left(t_{1}\right)-g\left(t_{0}\right)\right] } \\
& +\ldots+C\left(t-t_{n-1}\right)\left[g\left(t_{n-1}\right)-g\left(t_{n-2}\right)\right]+\left[g\left(t_{0}\right)-g\left(t_{n-1}\right)\right] .
\end{aligned}
$$

From this, using (5) and the Lipschitz condition for $g$, we obtain

$$
\left\|A u_{n}(t)\right\| \leq\|[C(t)-I] g(0)\|+L\left(M e^{\omega T}+1\right) t .
$$

On the other hand, for every $x^{*} \in D\left(A^{*}\right)$ we have

$$
\left\langle A u_{n}(t), x^{*}\right\rangle=\left\langle u_{n}(t), A^{*} x^{*}\right\rangle \rightarrow\left\langle v(t), A^{*} x^{*}\right\rangle .
$$

Since $D\left(A^{*}\right)$ is dense in $X^{*}$, from (10) and (11) it follows that $\left\{A u_{n}(t)\right\}$ is weakly convergent in $X$. Since $A$ is closed, it follows that $v(t) \in D(A)$, which completes the proof, $t$ being arbitrary in $[0, T]$.

Rem ark 1 . The inequality (10) leads to

(12) $\left\|A \int_{0}^{t} S(t-s) g(s) d s\right\| \leq\|[C(t)-I] g(0)\|+L\left(M e^{\omega T}+1\right) t, \quad t \in[0, T]$.

Lemma 2. Suppose $\left(\mathrm{Z}_{1}\right)$ and $\left(\mathrm{Z}_{2}\right)$. Let $x_{0} \in X$ be such that $S(t) x_{0} \in$ $D(A)$ for $t \in \mathbb{R}$ and the mapping $\mathbb{R} \ni t \rightarrow A S(t) x_{0}$ is locally bounded. Then $x_{0} \in E$.

Proof. Let

$$
h(t):=A S(t) x_{0}, \quad t \in \mathbb{R} .
$$

From the density of $D\left(A^{*}\right)$ in $X^{*}$, the strong continuity of $S$ with respect to $t \in \mathbb{R}$ and the local boundedness of $h$ it follows that $h$ is weakly continuous. By an argument similar to that of [6, Theorem IX.1] one can prove that $h$ is strongly measurable. Clearly $\|h\|$ is locally bounded. It follows that $h$ 
is Bochner integrable on every compact subset $[\alpha, \beta]$ of $\mathbb{R}$ and, in addition, that

$$
\left\|\int_{\alpha}^{\beta} h(t) d t\right\| \leq \int_{\alpha}^{\beta}\|h(t)\| d t
$$

Moreover, the function

$$
s \rightarrow \int_{\alpha}^{\beta} h(t+s) d t
$$

is continuous (in the norm topology), since $h$ is locally bounded.

Let $\alpha<\tau<\beta$ and $t_{0} \in \mathbb{R}$. Since

$$
\begin{aligned}
h\left(t_{0}\right) & =A S\left(t_{0}\right) x_{0}=A\left[2 C(\tau) S\left(t_{0}-\tau\right)-S\left(t_{0}-2 \tau\right)\right] x_{0} \\
& =2 C(\tau) h\left(t_{0}-\tau\right)-h\left(t_{0}-2 \tau\right),
\end{aligned}
$$

we have

$$
(\beta-\alpha) h\left(t_{0}\right)=\int_{\alpha}^{\beta} h\left(t_{0}\right) d \tau=2 \int_{\alpha}^{\beta} C(\tau) h\left(t_{0}-\tau\right) d \tau-\int_{\alpha}^{\beta} h\left(t_{0}-2 \tau\right) d \tau .
$$

From this we obtain

$$
\begin{aligned}
(\beta-\alpha)\left[h\left(t_{0}+\eta\right)-h\left(t_{0}\right)\right]= & 2 \int_{\alpha}^{\beta} C(\tau)\left[h\left(t_{0}+\eta-\tau\right)-h\left(t_{0}-\tau\right)\right] d \tau \\
& -\int_{\alpha}^{\beta}\left[h\left(t_{0}+\eta-2 \tau\right)-h\left(t_{0}-2 \tau\right)\right] d \tau .
\end{aligned}
$$

Therefore

$$
\begin{aligned}
(\beta-\alpha)\left\|h\left(t_{0}+\eta\right)-h\left(t_{0}\right)\right\| \leq & 2 \sup _{\alpha \leq \eta \leq \beta}\|C(\eta)\| \int_{t_{0}-\beta}^{t_{0}-\alpha}\|h(s+\eta)-h(s)\| d s \\
& +\frac{1}{2} \int_{t_{0}-2 \beta}^{t_{0}-2 \alpha}\|h(s+\eta)-h(s)\| d s .
\end{aligned}
$$

Approximating $h$ by finite-valued functions, we see that the right side of the last inequality tends to zero as $\eta \rightarrow 0$. We have thus proved that the mapping $\mathbb{R} \ni t \rightarrow h(t)$ is continuous (in the norm topology).

Now we have

$$
\begin{aligned}
& k^{-1}\left[C(t+k) x_{0}-C(t) x_{0}\right]=2 k^{-1} A S(k / 2) S(t+k / 2) x_{0} \\
& =2 k^{-1}[S(k / 2)-S(0)] h(t+k / 2) \rightarrow S^{\prime}(0) h(t) \quad \text { as } k \rightarrow 0 .
\end{aligned}
$$

From this we get

$$
C^{\prime}(t) x_{0}=h(t) \quad \text { for } t \in \mathbb{R} \text {. }
$$


From (14) and the continuity of $h$, the statement of Lemma 2 follows.

LEMMA 3. Under the assumptions of Lemma 1, the formula

$$
y(t):=\int_{0}^{t} C(t-s) g(s) d s, \quad t \in[0, T],
$$

defines a function from $[0, T]$ into $E$.

Proof. Fix $t \in[0, T]$. Using Lemma 2 it is enough to prove that $S(r) y(t) \in D(A)$ for $r \in \mathbb{R}$ and that the function $r \rightarrow A S(r) y(t)$ is locally bounded on $\mathbb{R}$. We have

$S(r) y(t)=S(r) \int_{0}^{t} C(t-s) g(s) d s=\frac{1}{2} \int_{0}^{t}[S(r+t-s)+S(r-t+s)] g(s) d s$.

From this by Lemma 1 we obtain $S(r) y(t) \in D(A)$. The inequality (12) implies that for any $-\infty<\alpha<\beta<\infty$ there exists a constant $K>0$ such that for all $r \in[\alpha, \beta]$ and $t \in[0, T]$

$$
\|A S(r) y(t)\| \leq K
$$

The proof of Lemma 3 is complete.

LEMMA 4. Under the assumptions of Lemma 1 , the function $w:[0, T] \rightarrow$ $X$ given by

$$
w(t):=A \int_{0}^{t} S(t-s) g(s) d s
$$

is continuous.

Proof. Fix $t \in[0, T]$ and let $t+\delta \in[0, T]$, where $\delta \neq 0$. We have

$$
\begin{aligned}
& w(t+\delta)-w(t) \\
& =A \int_{0}^{t}[S(t+\delta-s)-S(t-s)] g(s) d s+A \int_{t}^{t+\delta} S(t+\delta-s) g(s) d s \\
& =A S(\delta) \int_{0}^{t} C(t-s) g(s) d s+[C(\delta)-I] A \int_{0}^{t} S(t-s) g(s) d s \\
& \quad+A \int_{t}^{t+\delta} S(t+\delta-s) g(s) d s=w_{1}+w_{2}+w_{3} .
\end{aligned}
$$

We prove that $w_{i} \rightarrow 0$ as $\delta \rightarrow 0$ for $i=1,2,3$. Indeed, we have $w_{1}=$ $A S(\delta) \int_{0}^{t} C(t-s) g(s) d s=A S(\delta) y(t) \rightarrow 0$ as $\delta \rightarrow 0$, where $y(t)$ is defined by (15), and by Lemma $3, y(t) \in E$ for every $t \in[0, T]$. Next, $w_{2}=$ 
$[C(\delta)-I] A \int_{0}^{t} S(t-s) g(s) d s=[C(\delta)-I] w(t) \rightarrow 0$ as $\delta \rightarrow 0$. By (12) we get

$$
\begin{aligned}
\left\|w_{3}\right\|= & \left\|A \int_{t}^{t+\delta} S(t+\delta-s) g(s) d s\right\|=\left\|A \int_{0}^{\delta} S(\delta+s) g(t+s) d s\right\| \\
& \leq\|[C(\delta)-I] g(t)\|+L\left(M e^{\omega T}+1\right)|\delta| \rightarrow 0 \quad \text { as } \delta \rightarrow 0 .
\end{aligned}
$$

This completes the proof, $t$ being arbitrary in $[0, T]$.

3. Linear inhomogeneous problem corresponding to (1). In this section we consider the linear problem corresponding to (1), i.e.

$$
\left\{\begin{array}{l}
\frac{d^{2} u}{d t^{2}}=A u+g(t), \quad t \in(0, T] \\
u(0)=u_{0}, \quad \frac{d u}{d t}(0)=u_{1}
\end{array}\right.
$$

The motivation for this consideration comes from [4]. In [4] the authors consider the problem (18) under the assumption that the function $g: \mathbb{R} \rightarrow X$ is continuously differentiable. We only assume that $g$ satisfies the Lipschitz condition. The fundamental role will be played by assumption $\left(\mathrm{Z}_{2}\right)$.

Definition 1. A function $u:[0, T] \rightarrow X$ is called a solution of the problem (18) if it is of class $C^{1}$ in $[0, T], C^{2}$ in $(0, T]$ and satisfies (18) for $t \in[0, T]$.

TheOREM 1. Suppose $\left(\mathrm{Z}_{1}\right)$ and $\left(\mathrm{Z}_{2}\right)$. Assume $g$ satisfies the Lipschitz condition in $[0, T], u_{0} \in D(A)$ and $u_{1} \in E$. Then the problem (18) has exactly one solution $u$, given by

$$
u(t)=C(t) u_{0}+S(t) u_{1}+\int_{0}^{t} S(t-s) g(s) d s, \quad t \in[0, T] .
$$

Proof. Since the function $u(t)=C(t) u_{0}+S(t) u_{1}$ is the solution of the corresponding homogeneous equation with initial conditions $u(0)=u_{0}$ and $(d u / d t)(0)=u_{1}$, we need only show that the function $v$ defined by (8) satisfies the equation in (18) and the conditions $v(0)=0,(d v / d t)(0)=0$. From (8) and the strong continuity of a cosine family it follows that $v$ is continuously differentiable on $[0, T]$, and

$$
v^{\prime}(t):=\frac{d v}{d t}=\int_{0}^{t} C(t-s) g(s) d s, \quad t \in[0, T] .
$$

To prove our theorem it is enough to show that $v^{\prime}:[0, T] \rightarrow X$ is of class 
$C^{1}, v(0)=0$ and $v^{\prime}(0)=0$. We have

$v^{\prime}(t+h)-v^{\prime}(t)=\int_{0}^{t}[C(t+h-s)-C(t-s)] g(s) d s+\int_{t}^{t+h} C(t+h-s) g(s) d s$ for $t \in(0, T], h \neq 0$ and $t+h \in[0, T]$. Therefore

$$
\begin{aligned}
& h^{-1}\left[v^{\prime}(t+h)-v^{\prime}(t)\right]=h^{-1}[C(h)-I] \int_{0}^{t} C(t-s) g(s) d s \\
& \quad+h^{-1} S(h) A \int_{0}^{t} S(t-s) g(s) d s+h^{-1} \int_{t}^{t+h} C(t+h-s) g(s) d s \\
& =h^{-1}[C(h)-I] v^{\prime}(t)+h^{-1} S(h) w(t)+h^{-1} \int_{t}^{t+h} C(t+h-s) g(s) d s,
\end{aligned}
$$

where $w$ is given by (17). Since $v^{\prime}(t) \in E$ (see Lemma 3), we have

$$
\lim _{h \rightarrow 0} h^{-1}[C(h)-I] v^{\prime}(t)=0,
$$

$$
\begin{aligned}
\lim _{h \rightarrow 0} h^{-1} S(h) w(t) & =\lim _{h \rightarrow 0} h^{-1}[S(h)-S(0)] w(t)=C(0) w(t) \\
& =w(t)=A v(t)
\end{aligned}
$$

From the inequality

$$
\begin{aligned}
& \left\|h^{-1} \int_{t}^{t+h} C(t+h-s) g(s) d s-g(t)\right\|=\left\|h^{-1} \int_{0}^{h} C(r) g(t+h-r) d r-g(t)\right\| \\
& \leq\left\|h^{-1} \int_{0}^{h} C(r)[g(t+h-r)-g(t)] d r\right\|+\left\|h^{-1} \int_{0}^{h}[C(r)-I] g(t) d r\right\| \\
& \leq M L e^{\omega T}|h|+\sup \{\|[C(r)-I] g(t)\|:|r| \leq|h|\}
\end{aligned}
$$

it follows imediately that

$$
\lim _{h \rightarrow 0} h^{-1} \int_{t}^{t+h} C(t+h-s) g(s) d s=g(t) \quad \text { for } t \in(0, T] .
$$

By (21)-(23) we have

$$
v^{\prime \prime}(t)=A v(t)+g(t), \quad t \in(0, T] .
$$

In virtue of Lemma 4 , the function $w=A v$ is continuous in $(0, T]$. Consequently, $v \in C^{1}([0, T]) \cap C^{2}((0, T])$ and so $u \in C^{1}([0, T]) \cap C^{2}((0, T])$ where 
$u$ is given by (19). It is obvious that $v(0)=0$ and $v^{\prime}(0)=0$. This proves that $u$ is a solution of problem (18).

To show the uniqueness it sufices to remark that if $g:[0, T] \rightarrow X$ is continuous, $u:[0, T] \rightarrow X$ is twice continuously differentiable in $(0, T]$, $u(t) \in D(A)$ for $t \in[0, T]$ and $u$ satisfies (18), then $u$ is given by (19) (cf. [4, Proposition 2.4]). This completes the proof of Theorem 1.

\section{Existence and uniqueness of solution of problem (1)}

Definition 2. A function $u:[0, T] \rightarrow X$ is said to be a solution of the problem (1) if:

(i) $u$ is of class $C^{1}$ in $[0, T]$,

(ii) $u$ is $C^{2}$ in $(0, T]$,

(iii) $u(0)=u_{0}$ and $u^{\prime}(0)=u_{1}$,

(iv) $u^{\prime \prime}(t)=A u(t)+f\left(t, u(t), u^{\prime}(t)\right)$ for $t \in(0, T]$.

Similarly to the linear case we have the following theorem (cf. [4, Proposition 2.4]).

Theorem 2. If $f:[0, T] \times X \times X \rightarrow X$ is continuous and $u$ is a solution of the problem (1), then $u$ is a solution of the integral equation

$$
u(t)=C(t) u_{0}+S(t) u_{1}+\int_{0}^{t} S(t-s) f\left(s, u(s), u^{\prime}(s)\right) d s .
$$

Theorem 3. Suppose $\left(\mathrm{Z}_{1}\right)$ and $\left(\mathrm{Z}_{2}\right)$ and let $u_{0} \in D(A), u_{1} \in E$. Let $f:[0, T] \times X \times X \rightarrow X$ be a Lipschitzian mapping with Lipschitz constant $L>0$. If $u \in C^{1}([0, T], X)$ is a solution of the equation (25), then $u$ is a solution of the problem (1).

Proof. Firstly we prove that $u$ and $u^{\prime}$ satisfy the Lipschitz condition in $[0, T]$. Let $t$ and $t+h$ be any two points of $[0, T]$. We have

$$
\begin{aligned}
u(t+h)-u(t)= & C(t+h) u_{0}+S(t+h) u_{1} \\
& +\int_{0}^{t+h} S(t+h-s) f\left(s, u(s), u^{\prime}(s)\right) d s-C(t) u_{0}-S(t) u_{1} \\
& -\int_{0}^{t} S(t-s) f\left(s, u(s), u^{\prime}(s)\right) d s .
\end{aligned}
$$

Since $C(t) u_{0}+S(t) u_{1}$ is of class $C^{2}$ in $[0, T]$, there exist $C_{1}>0$ and $C_{2}>0$ such that (cf. [4])

$$
\begin{gathered}
\left\|[C(t+h)-C(t)] u_{0}+[S(t+h)-S(t)] u_{1}\right\| \leq C_{1}|h|, \\
\left\|\left[C^{\prime}(t+h)-C^{\prime}(t)\right] u_{0}+\left[S^{\prime}(t+h)-S^{\prime}(t)\right] u_{1}\right\| \leq C_{2}|h| .
\end{gathered}
$$


Hence

$$
\begin{aligned}
& \|u(t+h)-u(t)\| \\
& \leq C_{1}|h|+\| \int_{0}^{t} S(s)\left[f\left(t+h-s, u(t+h-s), u^{\prime}(t+h-s)\right)\right. \\
& \left.\quad-f\left(t-s, u(t-s), u^{\prime}(t-s)\right)\right] d s \| \\
& \quad+\left\|\int_{t}^{t+h} S(s) f\left(t+h-s, u(t+h-s), u^{\prime}(t+h-s)\right) d s\right\| \\
& \leq C_{1}|h|+\int_{0}^{t} M e^{\omega T} L(|h|+\|u(t+h-s)-u(t-s)\| \\
& \left.\quad+\left\|u^{\prime}(t+h-s)-u^{\prime}(t-s)\right\|\right) d s+M e^{\omega T} K|h|,
\end{aligned}
$$

where $K:=\sup \left\{\left\|f\left(t, u(t), u^{\prime}(t)\right)\right\|: t \in[0, T]\right\}$. From this we get

(26) $\quad\|u(t+h)-u(t)\|$

$$
\leq C_{3}|h|+C_{4} \int_{0}^{t}\left(\|u(s+h)-u(s)\|+\left\|u^{\prime}(s+h)-u^{\prime}(s)\right\|\right) d s .
$$

On the other hand, we have

$$
u^{\prime}(t)=C^{\prime}(t) u_{0}+S^{\prime}(t) u_{1}+\int_{0}^{t} C(t-s) f\left(s, u(s), u^{\prime}(s)\right) d s .
$$

From this we obtain analogously

(27) $\quad\left\|u^{\prime}(t+h)-u^{\prime}(t)\right\|$

$$
\leq C_{5}|h|+C_{6} \int_{0}^{t}\left(\|u(s+h)-u(s)\|+\left\|u^{\prime}(s+h)-u^{\prime}(s)\right\|\right) d s .
$$

The inequalities (26) and (27) lead to

(28) $\quad\|u(t+h)-u(t)\|+\left\|u^{\prime}(t+h)-u^{\prime}(t)\right\|$

$$
\leq \widetilde{C}|h|+\bar{C} \int_{0}^{t}\left(\|u(s+h)-u(s)\|+\left\|u^{\prime}(s+h)-u^{\prime}(s)\right\|\right) d s .
$$

By Gronwall's inequality (see e.g. [2]) we have

$$
\|u(t+h)-u(t)\|+\left\|u^{\prime}(t+h)-u^{\prime}(t)\right\| \leq C|h|,
$$

where $C>0$ is a constant. From (29) it follows that $u$ and $u^{\prime}$ satisfy the Lipschitz condition in $[0, T]$ with constant $C$. This implies that the mapping 
$[0, T] \ni t \rightarrow f\left(t, u(t), u^{\prime}(t)\right)$ also satisfies the Lipschitz condition. Thus, by Theorem $1, u$ is a solution of the equation

$$
\frac{d^{2} z}{d t^{2}}=A z+f\left(t, u(t), u^{\prime}(t)\right), \quad t \in(0, T],
$$

with the initial conditions

$$
z(0)=u_{0}, \quad z^{\prime}(0)=u_{1} .
$$

This means that $u$ is a solution of the problem (1).

Theorem 4. Suppose $\left(\mathrm{Z}_{1}\right)$ and $\left(\mathrm{Z}_{2}\right)$. Let $f:[0, T] \times X \times X \rightarrow X$ be continuous. Suppose that there exists $L>0$ such that

$\|f(t, x, y)-f(t, u, v)\| \leq L(\|x-u\|+\|y-v\|) \quad$ for $t \in[0, T], x, y, u, v \in X$.

Then for any $u_{0} \in E$ and $u_{1} \in X$ there exists exactly one solution of the integral equation (25) belonging to $C^{1}([0, T], X)$.

Proof. It is a slight modification of the proof of Theorem 4.5 of [5]. Let $C:=\sup \{\|C(t)\|+\|S(t)\|: t \in[0, T]\}$. In the space $C^{1}([0, T], X)$ consider the two norms

$$
\begin{aligned}
\|u\|_{1} & :=\sup \left\{\|u(t)\|+\left\|u^{\prime}(t)\right\|: t \in[0, T]\right\}, \\
|u|_{1} & :=\sup \left\{e^{-C L t}\left(\|u(t)\|+\left\|u^{\prime}(t)\right\|\right): t \in[0, T]\right\} .
\end{aligned}
$$

Since $|u|_{1} \leq\|u\|_{1} \leq e^{C L T}|u|_{1}$, these norms are equivalent.

Let

$$
\begin{array}{r}
(G u)(t):=C(t) u_{0}+S(t) u_{1}+\int_{0}^{t} S(t-s) f\left(s, u(s), u^{\prime}(s)\right) d s \\
\quad t \in[0, T] .
\end{array}
$$

By the hypotheses, it is evident that (32) defines an operator from $C^{1}([0, T], X)$ into itself. Thus Theorem 4 will be proved if we show that $G$ is contractive under the norm (31). We have

$$
\begin{aligned}
\|(G u)(t)-(G v)(t)\| & =\left\|\int_{0}^{t} S(t-s)\left[f\left(s, u(s), u^{\prime}(s)\right)-f\left(s, v(s), v^{\prime}(s)\right)\right] d s\right\| \\
& \leq \int_{0}^{t}\|S(t-s)\| L\left(\|u(s)-v(s)\|+\left\|u^{\prime}(s)-v^{\prime}(s)\right\|\right) d s
\end{aligned}
$$


and

$$
\begin{aligned}
\left\|(G u)^{\prime}(t)-(G v)^{\prime}(t)\right\| & \\
& =\left\|\int_{0}^{t} C(t-s)\left[f\left(s, u(s), u^{\prime}(s)\right)-f\left(s, v(s), v^{\prime}(s)\right)\right] d s\right\| \\
& \leq \int_{0}^{t}\|C(t-s)\| L\left(\|u(s)-v(s)\|+\left\|u^{\prime}(s)-v^{\prime}(s)\right\|\right) d s .
\end{aligned}
$$

Therefore

$$
\begin{aligned}
|G u-G v|_{1} \leq & \sup \left\{e^{-C L t} L \int_{0}^{t}(\|S(t-s)\|+\|C(t-s)\|)\right. \\
& \left.\times\left(\|u(s)-v(s)\|+\left\|u^{\prime}(s)-v^{\prime}(s)\right\|\right) d s: t \in[0, T]\right\} \\
\leq & \sup \left\{e^{-C L t} C L \int_{0}^{t} e^{-C L s}\right. \\
& \left.\times e^{C L s}\left(\|u(s)-v(s)\|+\left\|u^{\prime}(s)-v^{\prime}(s)\right\|\right) d s: t \in[0, T]\right\} \\
\leq & C L \sup \left\{e^{-C L t}|u-v|_{1} \int_{0}^{t} e^{C L s} d s: t \in[0, T]\right\} \\
= & C L|u-v|_{1} \sup \left\{e^{-C L t} \frac{1}{C L}\left(e^{C L t}-1\right): t \in[0, T]\right\} \\
\leq & \left(1-e^{-C L T}\right)|u-v|_{1} \leq \alpha|u-v|_{1},
\end{aligned}
$$

where $\alpha:=1-e^{-C L T}<1$ is a constant independent of $t$. Theorem 4 is proved.

As a consequence of Theorems 3 and 4 we get

THEOREM 5. If

(i) the operator $A$ satisfies assumptions $\left(\mathrm{Z}_{1}\right)$ and $\left(\mathrm{Z}_{2}\right)$,

(ii) $f:[0, T] \times X \times X \rightarrow X$ satisfies the Lipschitz condition,

(iii) $u_{0} \in D(A)$ and $u_{1} \in E$,

then the problem (1) has a unique solution which is the unique solution of the integral equation (25). 


\section{References}

[1] T. Kato, Perturbation Theory for Linear Operators, Springer, 1966.

[2] R. Rabczuk, Elements of the Theory of Differential Inequalities, PWN, Warszawa 1976 (in Polish).

[3] I. Segal, Nonlinear semi-groups, Ann. of Math. 78 (1963), 339-364.

[4] C. C. Travis and G. F. Webb, Cosine families and abstract nonlinear second order differential equations, Acta Math. Acad. Sci. Hungar. 32 (1978), 75-96.

[5] T. Winiarska, Differential Equations with Parameter, Monograph 68, Technical University of Cracow, 1988.

[6] K. Yosida, Functional Analysis, Springer, New York 1980.

\section{INSTITUTE OF MATHEMATICS}

TECHNICAL UNIVERSITY OF CRACOW

WARSZAWSKA 24

31-155 KRAKÓW, POLAND

Reçu par la Rédaction le 6.12.1989

Révisé le 11.6.1990 